Article

\title{
Resource-Efficient Pet Dog Sound Events Classification Using LSTM-FCN Based on Time-Series Data
}

\author{
Yunbin Kim, Jaewon Sa $₫$, Yongwha Chung, Daihee Park and Sungju Lee * \\ Department of Computer Convergence Software, Korea University, Sejong City 30019, Korea; \\ kyb2629@korea.ac.kr (Y.K.); sjwon92@korea.ac.kr (J.S.); ychungy@korea.ac.kr (Y.C.); dhpark@korea.ac.kr (D.P.) \\ * Correspondence: peacfeel@korea.ac.kr; Tel.: +82-44-860-1373
}

Received: 21 September 2018; Accepted: 15 November 2018; Published: 18 November 2018

\begin{abstract}
The use of IoT (Internet of Things) technology for the management of pet dogs left alone at home is increasing. This includes tasks such as automatic feeding, operation of play equipment, and location detection. Classification of the vocalizations of pet dogs using information from a sound sensor is an important method to analyze the behavior or emotions of dogs that are left alone. These sounds should be acquired by attaching the IoT sound sensor to the dog, and then classifying the sound events (e.g., barking, growling, howling, and whining). However, sound sensors tend to transmit large amounts of data and consume considerable amounts of power, which presents issues in the case of resource-constrained IoT sensor devices. In this paper, we propose a way to classify pet dog sound events and improve resource efficiency without significant degradation of accuracy. To achieve this, we only acquire the intensity data of sounds by using a relatively resource-efficient noise sensor. This presents issues as well, since it is difficult to achieve sufficient classification accuracy using only intensity data due to the loss of information from the sound events. To address this problem and avoid significant degradation of classification accuracy, we apply long short-term memory-fully convolutional network (LSTM-FCN), which is a deep learning method, to analyze time-series data, and exploit bicubic interpolation. Based on experimental results, the proposed method based on noise sensors (i.e., Shapelet and LSTM-FCN for time-series) was found to improve energy efficiency by 10 times without significant degradation of accuracy compared to typical methods based on sound sensors (i.e., mel-frequency cepstrum coefficient (MFCC), spectrogram, and mel-spectrum for feature extraction, and support vector machine (SVM) and k-nearest neighbor (K-NN) for classification).
\end{abstract}

Keywords: pet dogs; separation anxiety; IoT sensor; sound events processing; resource efficiency; LSTM-FCN

\section{Introduction}

Research on processing and analyzing big data in the IoT (Internet of Things) field has attracted considerable attention lately. In particular, parallel processing techniques, cloud computing technology, research for providing real-time services to users, and encryption have been actively investigated to find ways to more efficiently process large amounts of data [1-5]. Recently, an increase in the number of single-person households has led to studies on the behavior and control of companion animals, specifically the use of IoT sensor technology for the management of pet dogs. Research has been conducted on detecting dog behavior like sitting, walking, or running, to identify behavioral states [6] and actions like barking, growling, howling, or whining to identify emotional states [7]. For example, one study was conducted on pet dog health management that involved the detection of pet dog behaviors by means of acceleration sensors and heart rate sensors to identify food intake and/or 
bowel movements. Techniques have been developed for analyzing pet dog behavior to understand the emotional states, like depression or separation anxiety, of pet dogs who spend their time alone at home. With regard to understanding the emotions of pet dogs, sound events provide the most important information, which is why sound sensors are widely used [8]. In general, to classify the sound events, sound data are acquired by sound sensors, pre-processed in various ways to perform tasks like feature extraction, and then classified. Because data transfer and battery consumption are major issues for such sensors (sound and transmission sensors), which tend to have limitations on their capabilities, we need a way to classify pet dog sound events for resource-limited sensor devices.

In this paper, we propose a way to efficiently classify pet dog sound events using intensity data and long short-term memory-fully convolutional networks (LSTM-FCN) based on time-series data. For this purpose, we acquire only intensity data by using a relatively resource-efficient noise sensor. In other words, the intensity data is acquired with an attachable noise sensor placed on a pet dog, and intensity sequences corresponding to barking, growling, howling, and whining are classified using a time-series data analysis method. It is difficult to attain sufficient classification accuracy using intensity data alone due to the loss of other information compared to sound data. To avoid significant degradation of classification accuracy, we apply LSTM-FCN, which is a method of deep learning analysis based on time-series data, and exploit the idea of bicubic interpolation.

To verify the proposed method, actual pet dog sound events corresponding to barking, growling, howling, and whining were acquired from the internet, and the database was constructed using ground truth labels. Experimental results show that the proposed method based on noise sensor (i.e., Shapelet [9] and LSTM-FCN [10] for time-series) improves energy efficiency by 10 times without significant degradation of accuracy performance compared to typical methods based on sound sensor (i.e., MFCC [11], spectrogram [12], and mel-spectrum [13] for feature extraction, and SVM [14] and K-NN [15] for classification).

This paper is organized as follows. In Section 2, we summarize the time-series data classification problem and the LSTM-FCN approach that comprise the background of the proposed method. In Section 3, data acquisition, processing, and classification for intensity data obtained from attachable noise sensors are presented in detail. In Section 4, the experimental results are presented in terms of accuracy and energy efficiency. Finally, Section 5 discusses conclusions and future research.

\section{Background}

\subsection{Time-Series Classification}

Time-series data can confirm trends in data between the past and the present, and time-series data is also sensitive to time-based information. Time-series data is largely found in domains that utilize real-time sensor data $[16,17]$ such as traffic conditions $[18,19]$, speech recognition $[20,21]$, and weather information [22,23] using prediction and classification models [16-23]. In particular, a large amount of data flows from the sensor, and data warehouse technology [24] and techniques for analyzing this type of data are being developed. It is essential to convert the data into a meaningful form for accurate data analysis, which requires pre-processing the data before it can be used to develop a prediction or classification model. To improve classification accuracy, dimensional reduction [25-27] and data augmentation [28-30] have been studied. Garcke et al. [25] proposed a method to reduce the dimension of nonlinear time-series data extracted from wind turbines, setting the baseline so as to distinguish normal turbines from abnormal turbines, and monitoring the state of the wind turbines. In order to solve the multidimensional problem presented by time-series data acquired from a virtual sensor, dimension reduction was performed. In addition, Um et al. [28] proposed a method for applying convolutional neural networks (CNNs) to Parkinson's disease data acquired from wearable sensors. To overcome the issue of using only a small amount of data, they improved classification accuracy by using various data augmentation methods like jittering, scaling, and rotation. In this way, dimension adjustment of the data is performed to compensate for missing data, to solve 
the "overfitting problem" in which accuracy is reduced due to excessive amounts of training data, and to address the "underfitting problem".

\section{2. $L S T M-F C N$}

Time-series data is used in various fields to solve classification problems. Selecting a good classification model is important, as is acquiring high-quality data. Machine learning techniques such as hidden Markov models [31], dynamic time warping [32], and shapelets were developed to solve the time-series classification problem. LSTM-FCN is a recently developed method proposed by Karim et al. [10] to solve the time-series data classification problem.

It consists of two blocks, a fully convolutional block and LSTM block, which receive the same time-series data. We use three convolutional layers composed of temporal convolutions to extract the characteristics of the input time-series data, and use batch normalization and the ReLU activation function to avoid vanishing gradients and exploding gradients during the learning process. Simultaneously, the LSTM block performs a dimensional shuffle on the received time-series data to convert it into a multivariate time-series with a single time step, which is processed by the LSTM layer. Finally, the multivariate time-series processed in each block is connected to a softmax classification layer, in which the time-series data can be classified.

In this paper, we acquired the intensity of sounds from the pet dogs using an attachable noise sensor. Each sound event was labeled as four sound event classes. In this case, since the sound event consisted of a sequence of varying dimensions, the dimension of the intensity data was transformed uniformly, and normalization and interpolation were performed to make the standard deviation of the values constant. To solve the problem, we apply LSTM-FCN to distinguish the time-series data after pre-processing each sound event.

\section{Proposed Methods}

Wearable devices for pet dogs require continuous data acquisition, since the behavior of the dog in the home is not limited to a certain period of time. In this paper, we propose a classification method for pet dog sound events using intensity data acquired by a relatively resource-efficient noise sensor (LM-393). The acquired data is time-series data in which the observation exhibits a pattern of temporal order. The data is labeled as the following four sound event classes: barking, growling, howling, and whining. After the acquired intensity data is transmitted over the wireless network via the IoT platform, classification is performed via pre-processing and feature extraction through the following four operations:

1. Labeling sound event as barking, growling, howling, or whining.

2. Applying normalization methods to obtain a constant data distribution.

3. Extending the dimension of learning data by interpolation.

4. Applying the LSTM-FCN model to classify the pet dog sound events.

Figure 1 shows the overall structure of the proposed method. 


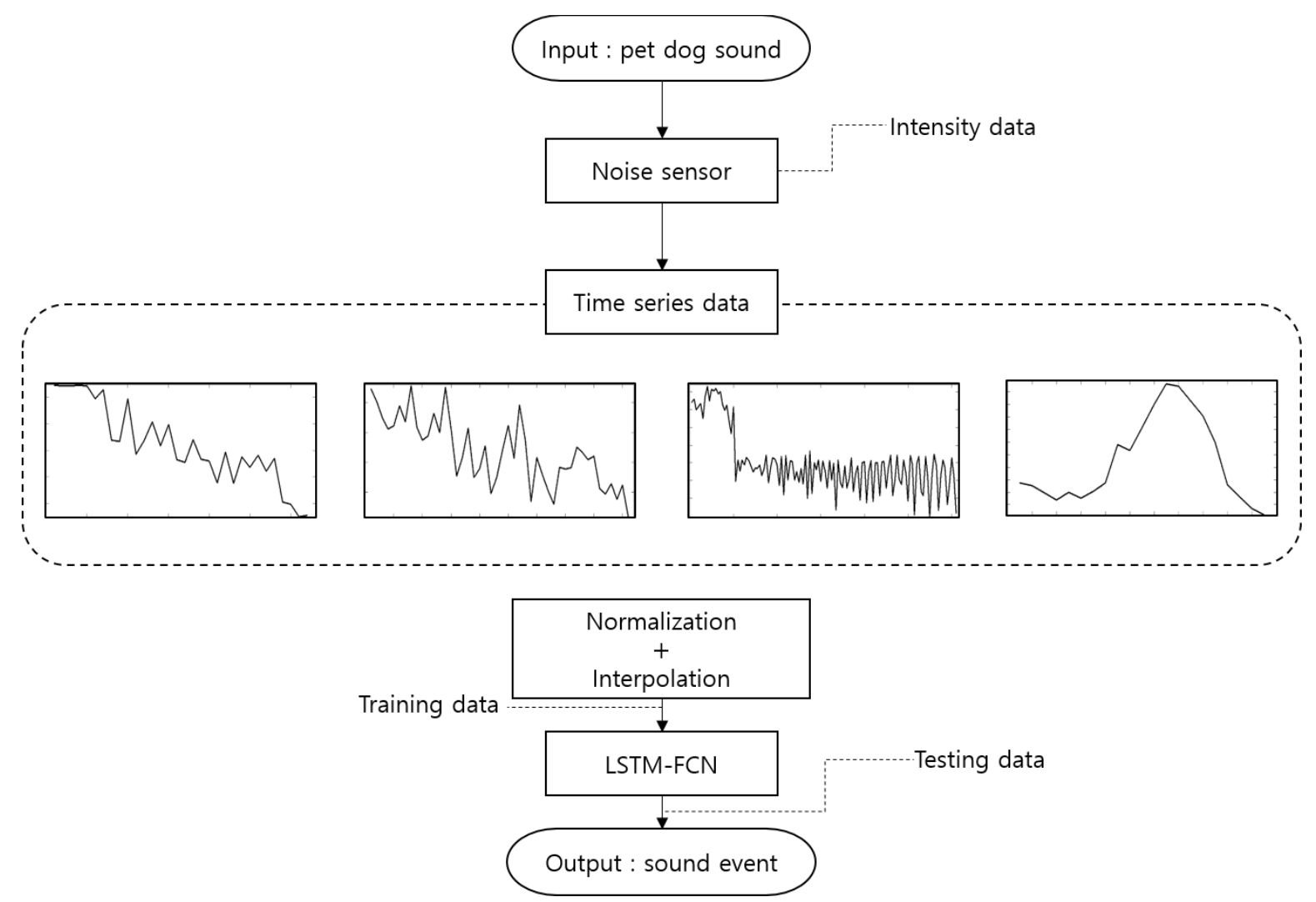

Figure 1. Overall structure of the proposed method.

\subsection{Pet Dog Sound Event Intensity Data Acquired by Noise Sensor}

In this paper, intensity data corresponding to pet dog sound events are acquired using a noise sensor (LM-393) integrated with an Arduino sensor module. The noise sensor can amplify and control the sound generated by means of a variable resistor located on the upper portion of the sensor, if the sensitivity of the sound intensity is lower than desired. It senses sound based on this sensitivity and outputs it as voltage. The size of the sensor is $32 \mathrm{~mm} \times 17 \mathrm{~mm} \times 1 \mathrm{~mm}$ and the voltage is $3.3 \mathrm{~V}$ or $5 \mathrm{~V}$.

A wireless noise sensor is attached to the neck of the pet dog to obtain intensity data. When attaching such a noise sensor, the sensor and the dog's neck strap must be finely adjusted to minimize noise caused by movement of the dog. The noise sensor outputs the intensity data over time at a rate of 138 data samples per second. The acquired intensity data is transmitted to the data storage device through Wi-Fi, after which each event is labeled as barking, growling, howling, or whining.

Figure 2 shows a noise sensor attached to the neck of a pet dog to acquire intensity data. 


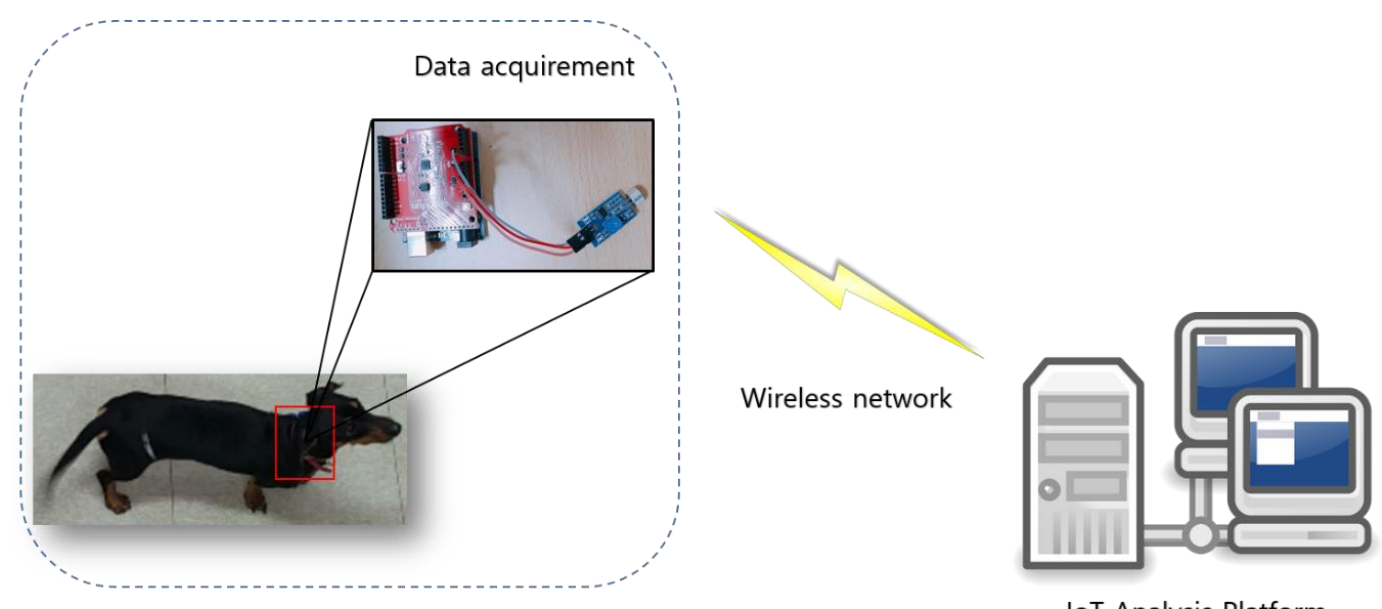

Figure 2. Intensity data acquisition using noise sensor. The noise sensors are used to collect intensity data and the collected intensity data is transmitted over the wireless network to the IoT analysis platform to process the data.

\subsection{Analysis of Pet Dog Sound Intensity}

Figure 3 shows plots of the sound data with the four sound features extracted from a sound sensor. When each sound event occurs (i.e., barking, growling, howling, or whining) the interval is set and extracted.

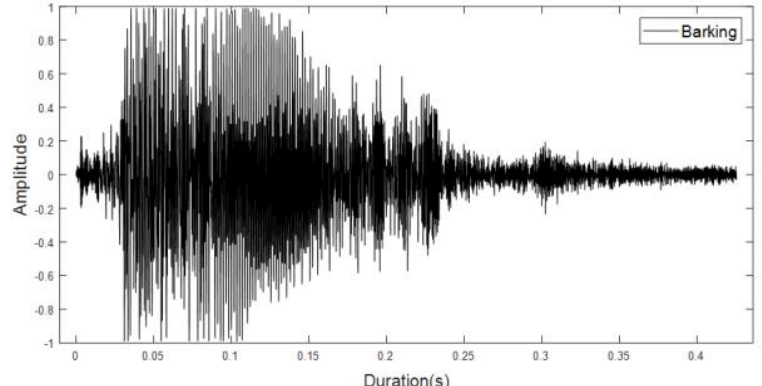

(a)

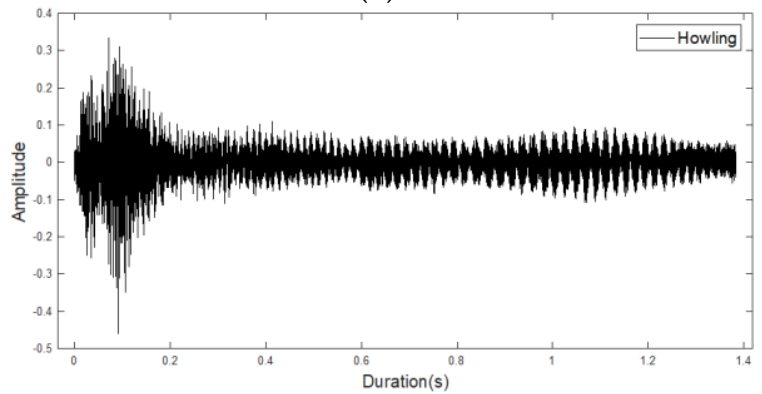

(c)

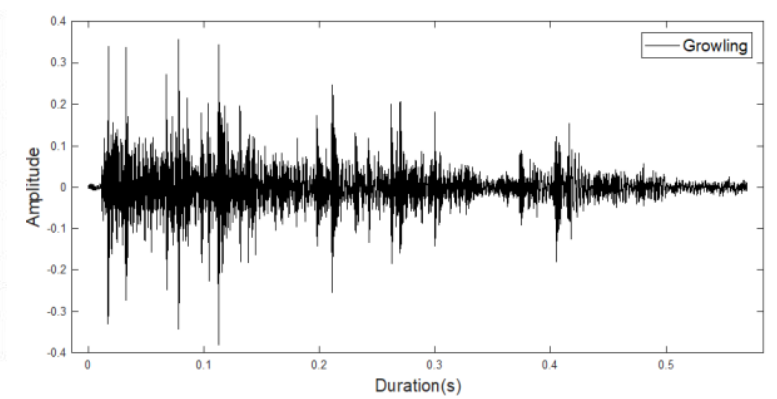

(b)

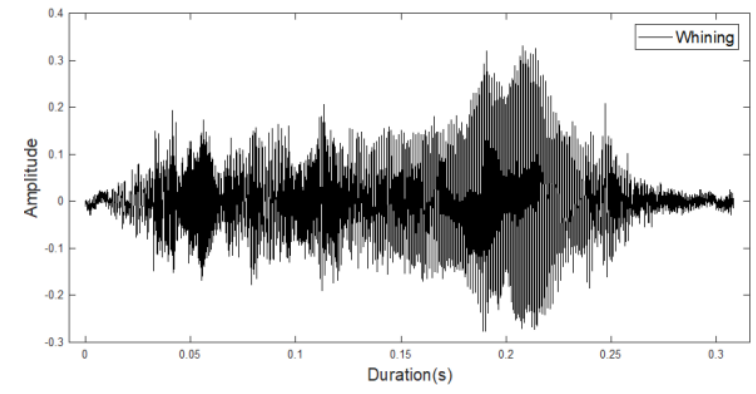

(d)

Figure 3. Waveforms for four pet dog sound events: (a) barking; (b) growling; (c) howling; (d) whining events.

In the waveforms, we can see that the four classes have different characteristics. Figure 3a is the data corresponding to a general barking sound, and illustrates the frequency for approximately $0.4 \mathrm{~s}$. Figure $3 b$ is the "growling sound", which exhibits a continuous signal. Figure $3 c$ is characteristic of "howling sound", which is a behavior by which pet dogs express loneliness. A strong waveform can be seen at the beginning of the sound, which decreases in the latter part. Figure $3 \mathrm{~d}$ represents "whining sound", a behavior that expresses fear and obedience, and is a representative example of howling to express separation anxiety. This exhibits a pattern similar to that of barking, but the amplitude is 
relatively low and the duration of the feature is short, with duration of approximately $0.1 \mathrm{~s}$. Table 1 shows information on the sound data in Figure 3. Each field includes "CM" (CompressionMethod), which refers to the compression method used, "NC" (NumChannels), which is the number of audio channels encoded in the audio file, and "SR" (SampleRate), the sample rate of the audio data contained in the file. Additionally, the total number of samples "TS" (TotalSamples), the file playback time "Duration", and the number of bits per sample "BPS" (BitsPerSample) encoded in the audio file are also included.

Note that the intensity data can be extracted from the sound data by treating the features as time-series data representing voltage information with the passage of time. At this time, the transmission option has 8 data bits, with the parity bit being set to "N", and the stop bit being set to 1 . Data is acquired at a rate of 138 samples per second.

Table 1. Information on pet dog sound data file format.

\begin{tabular}{clccccc}
\hline \multirow{2}{*}{ Pet Dog Sound Events } & \multicolumn{5}{c}{ Field Name } \\
\cline { 2 - 7 } & CM & NC & SR & TS & Duration & BPS \\
\hline Barking & Uncompressed & 1 & 22,050 & 5327 & 0.24 & 16 \\
Growling & Uncompressed & 1 & 22,050 & 11,461 & 0.51 & 16 \\
Howling & Uncompressed & 1 & 22,050 & 32,628 & 1.47 & 16 \\
Whining & Uncompressed & 1 & 22,050 & 6311 & 0.28 & 16 \\
\hline
\end{tabular}

Although the LM-393 cannot provide exact sound intensity as well as sound data, the sound intensity level can be obtained by calculating the sound amplitude through "Peak to Peak" which means the minimum and maximum value among the changing voltages from the diaphragm. The diaphragm acquires electrical signal (analog voltage signal) with change of air pressure for sound in the audible frequency range. Finally, the continuous analog voltage signal is converted into digital data by using ADC (Analog to Digital Converter) with sampling, quantization, and encoding. Note that the ADC is built into the noise sensor, and the resolution is 10 bits (i.e., $2^{10}=1024$ ). Therefore, the noise sensor divides voltage signal from GND (Ground, 0 V) to VCC (Voltage of Common Collector, $5 \mathrm{~V}$ ) by 10 bits resolution. The peak to peak represents the difference between these resolution ranges ( 0 to 1023), and then the calculated peak to peak data is converted to a value from 0 to $5 \mathrm{~V}$ (i.e., intensity level). In other words, with noise sensor, the intensity of the sound is measured by 1024 level (i.e., 1024 intensity level) with a value between 0 and $5 \mathrm{~V}$ [33].

In Figure 4, left figures show the intensity from the sound data, and right figures shows the intensity level (i.e., noise intensity) obtained from a noise sensor. Although the noise sensor can measure the intensity level, it is difficult to obtain the accurate original intensity (i.e., sound intensity) as shown in Figure 4. To solve the problem, we exploited the idea of the interpolation technique without the difference of result in a significant change in the overall data. To compare sound intensity and noise intensity, we represent the intensity level as dB units, as shown in Figure 4.
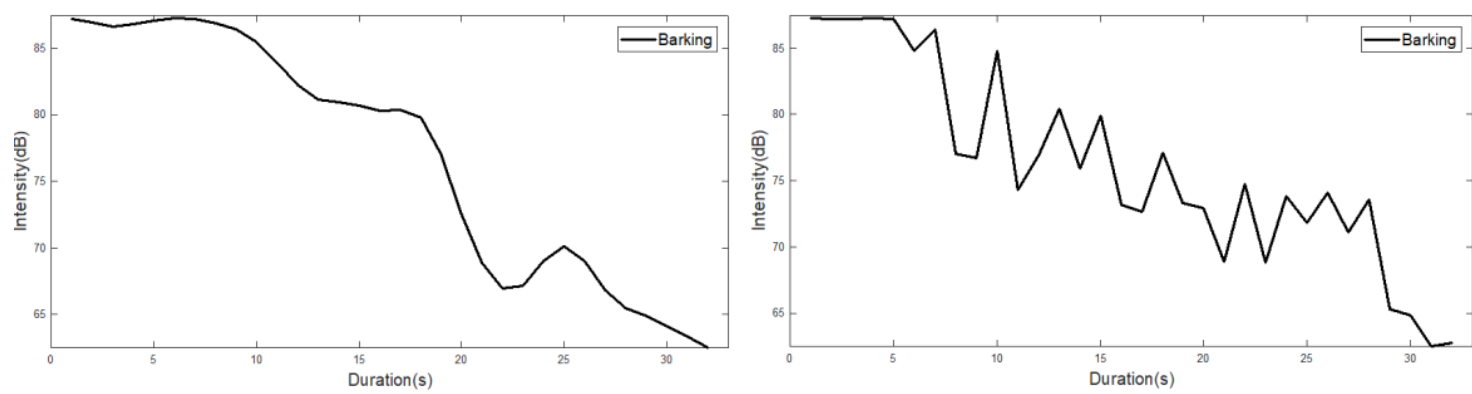

(a)

Figure 4. Cont. 

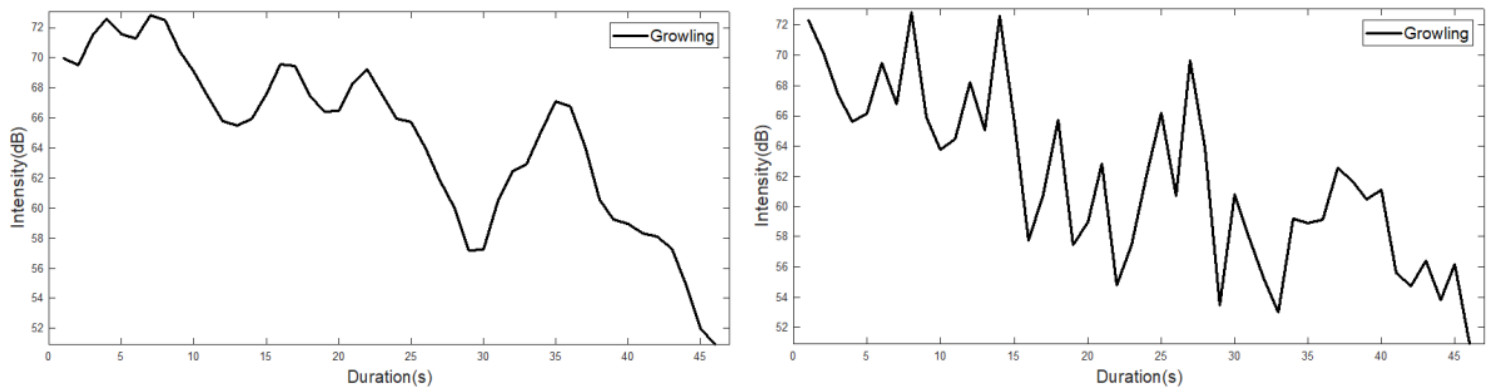

(b)
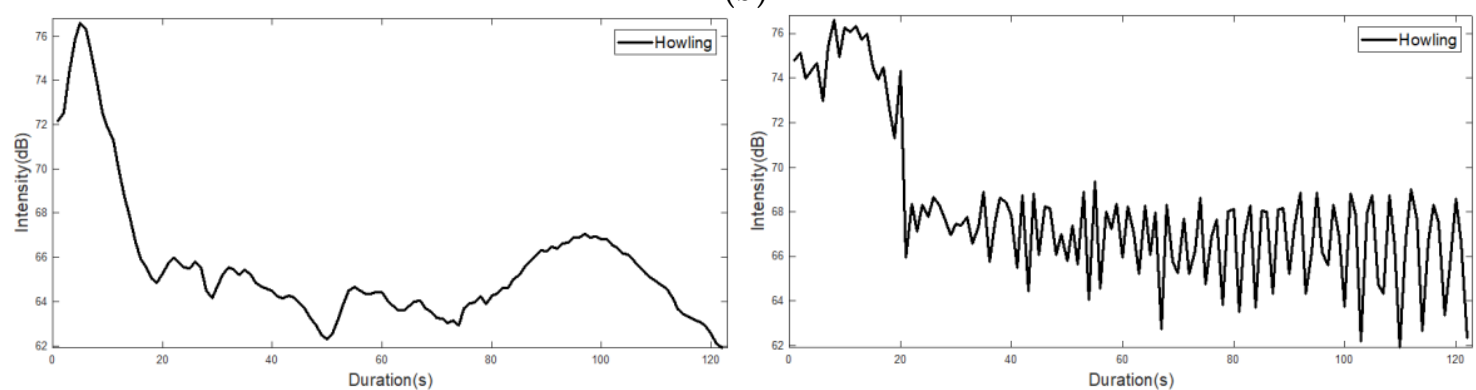

(c)
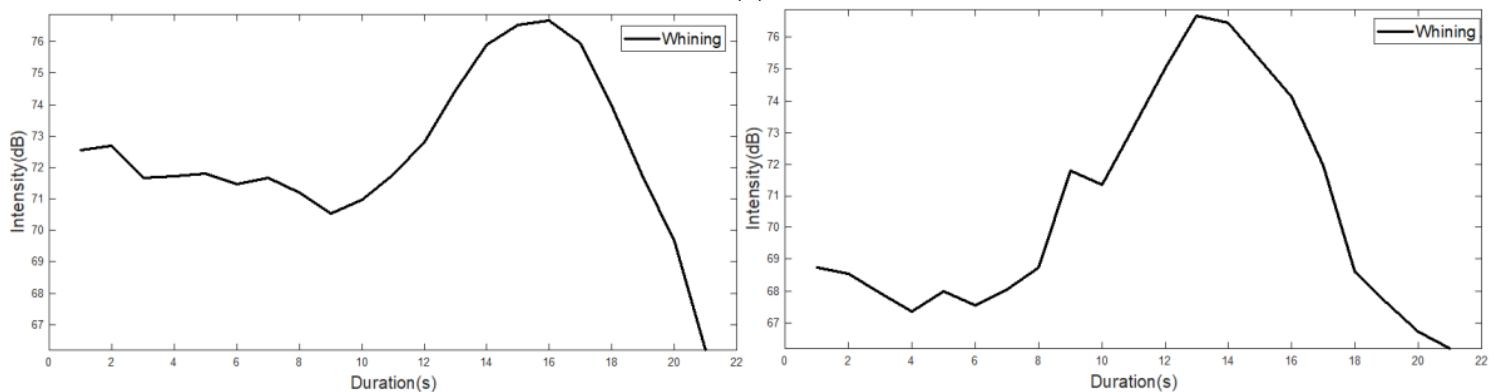

(d)

Figure 4. Intensity from the sound data and intensity level obtained from a noise sensor: (left) the intensity from the sound data; (right) the intensity level. (a) a barking event has a relatively short duration, and the value decreases rapidly after a certain period; (b) a growing event has a longer duration than the barking event, and also has a jagged characteristic; (c) a howling event shows the longest duration among the four sound events. It shows that the value of the early event is high and the value becomes low toward the rear part; (d) a whining event, such as barking, shows a short duration, and it also displays a jagged characteristic momentarily.

Figure 4 shows that the intensity level is shows a similar shape compared to the intensity. To find out the difference of intensity (i.e., sound sensor) and intensity level (i.e., noise sensor), we calculate the root mean square error (RMSE) with Equation (1), which is a generally used to measure the differences between values predicted by a model and the values observed. In Equation (1), $y_{i}$ and $\hat{y}_{i}$ are intensity and intensity level, respectively. Note that, the square root of the arithmetic average of the squared residuals of $y_{i}$ and $\hat{y}_{i}$ is statistically a standard deviation.

$$
\operatorname{RMSE}=\sqrt{\frac{1}{n} \sum_{i=1}^{n}\left(y_{i}-\hat{y}_{i}\right)^{2}}
$$

Table 2 shows the results of RMSE between intensity and intensity level. With decreased RMSE, the similarity of sound intensity and noise intensity is increased. As shown in Table 2 with comparison of intensity (i.e., sound sensor) and intensity level (i.e., noise sensor), The RMES results of same sound events is relatively lower (i.e., barking-barking, growling-growling, howling-howling, and whining-whining are 4.61, 4.70, 3.54, and 3.13, respectively) than the different sound events (i.e., 
barking with growling, howling, and whining are 14.79, 8.63, 13.57, respectively). Therefore, the noise sensor can measure the intensity level, even if it is difficult to obtain the accurate original intensity.

Table 2. The results of RMSE between intensity and intensity level.

\begin{tabular}{lccccc}
\hline \multirow{2}{*}{ Pet dog Sound Events } & \multicolumn{3}{c}{ Noise Sensor (Intensity Level) } \\
\cline { 3 - 5 } & & Barking & Growling & Howling & Whining \\
\hline \multirow{3}{*}{ Sound sensor (Intensity) } & Barking & 4.61 & 14.79 & 8.63 & 13.57 \\
& Growling & 10.41 & 4.70 & 8.14 & 8.34 \\
& Howling & 8.89 & 8.89 & 3.54 & 7.93 \\
& Whining & 9.57 & 8.38 & 7.81 & 3.13 \\
\hline
\end{tabular}

The samples of intensity data acquired from the noise sensor have different lengths from the beginning to the end of the sound event. The length of the data can be used as a criterion in the feature extraction process, and if the data length is short, it may cause underfitting of the data. Table 3 shows the minimum, maximum, mean, and median lengths of the intensity data for each sound event.

Table 3. The minimum, maximum, mean, and median lengths of the intensity data for each sound events.

\begin{tabular}{ccccc}
\hline \multirow{2}{*}{ Pet dog Sound Events } & \multicolumn{4}{c}{ Field Name } \\
\cline { 2 - 5 } & Minimum Length & Maximum Length & Mean Length & Median Length \\
\hline Barking & 5 & 47 & 19.24 & 19 \\
Growling & 16 & 405 & 59.59 & 56 \\
Howling & 51 & 646 & 188.60 & 161 \\
Whining & 5 & 198 & 27.97 & 19 \\
\hline
\end{tabular}

The minimum length in Table 3 shows that both barking and whining sound events have a length of 5. The barking sound event has the lowest arithmetic mean at 19.24. The barking and whining sound events, which have relatively short lengths, experience considerable data loss relative to the original sound data. As described above, short data lengths present difficulties in extracting features to solve the classification problem.

Furthermore, since the sounds of pet dogs are different in size at the same sound event, the characteristics of size should be judged pointless. There is a problem that the range of the value is not constant because the intensity data acquired from the noise sensor outputs the value of the voltage by measuring the sound amplitude. This problem can lead to confusion by judging the magnitude of the value as minimum, maximum, mean, and median of intensity data. Table 4 shows the size comparison of the values of all the data sets acquired from the sensor.

Table 4. Differences of voltage value according to each sound event of data extracted from sensor.

\begin{tabular}{ccccc}
\hline \multirow{2}{*}{ Pet Dog Sound Events } & \multicolumn{4}{c}{ Field Name } \\
\cline { 2 - 5 } & Minimum Voltage & Maximum Voltage & Mean Voltage & Median Voltage \\
\hline Barking & 0.98 & 25.39 & 15.70 & 17.58 \\
Growling & 0.98 & 8.79 & 6.29 & 5.86 \\
Howling & 0.98 & 11.72 & 7.68 & 7.81 \\
Whining & 0.98 & 13.67 & 8.32 & 7.81 \\
\hline
\end{tabular}

To solve this problem, the ranges must be equal and the distributions must be similar. In this paper, we apply 0-1 normalization to achieve this. By using the maximum and minimum values of the voltage time-series data, the data can be transformed into a data set having an average distribution between 0 and 1. Equation (2) represents $0-1$ normalization. 


$$
\text { Voltage }=\frac{x_{\text {voltage }}-\min \left(x_{\text {voltage }}\right)}{\max \left(x_{\text {voltage }}\right)-\min \left(x_{\text {voltage }}\right)}
$$

\subsection{Bicubic Interpolation}

In this paper, we apply anti-aliasing and interpolation to increase the data length without changing the features of the data. Interpolation is one of the image processing techniques used to acquire missing values among pixels when enlarging or reducing images. Especially, bicubic interpolation can be used in signal processing as well as image processing. It is performed by multiplying the values of the 16 adjacent vectors with weights based on their distance. This is advantageous in that interpolation can be performed naturally and accurately by obtaining the slope of the peripheral value and sampling the data. Bicubic interpolation is applied to the dataset obtained from the noise sensor to increase the amount of data by a factor of three. Equation (3) represents the process of bicubic interpolation:

$$
f(x, y)=\sum_{i=0}^{3} \sum_{j=0}^{3} a_{i j} x^{i} x^{i}
$$

Figure 5 shows the change in the length of the time-series data after bicubic interpolation. It can be confirmed that the additional data produced by the bicubic interpolation demonstrates no meaningful change compared to the original data.

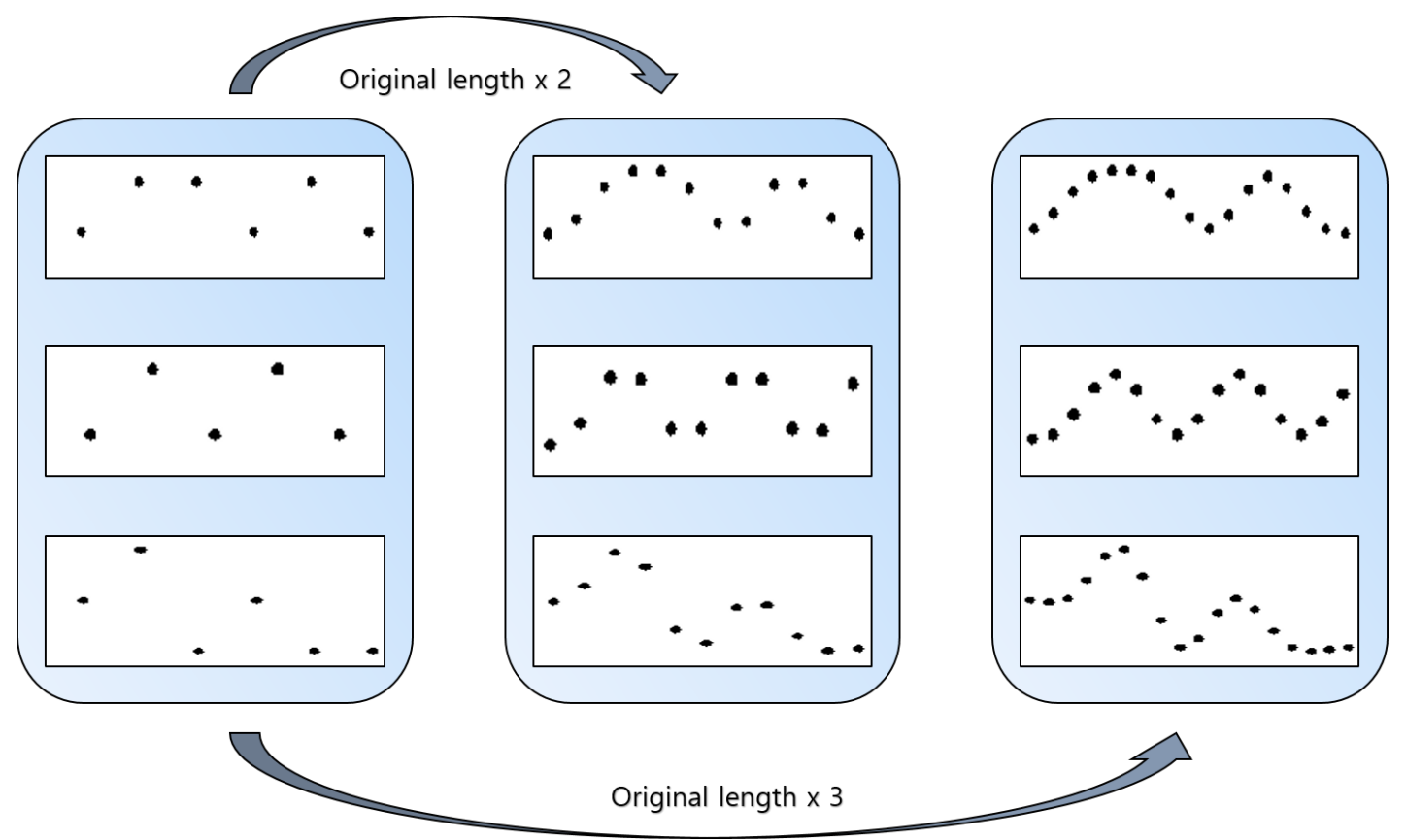

Figure 5. Sound events of increased length obtained via bicubic interpolation.

\subsection{Classification of Pet Dog Sound Events Using LSTM-FCN}

In this paper, we acquired intensity data for barking, growling, howling, and whining of pet dogs. The data were refined via bicubic interpolation, which is a traditional interpolation technique, and 0-1 normalization. We applied the LSTM-FCN method, which processes the input data through two networks, connects their results, and applies the softmax function. Seventy percent and $30 \%$ of the whole data were used in the learning process and evaluation process, respectively, of the LSTM-FCN. In other words, in 1200 intensity data samples, 840 comprised the training set, and the remaining 360 were used for the test set to verify the model. 
Figure 6 shows the application of the LSTM-FCN model to the voltage time-series pet dog sound data. The filter sizes of the convolution layers were set to 128, 256, and 128, respectively, by default, and the ReLU activation function was used. The initial batch size was 128, the number of classes was 4 , the maximum dimension was 647 , and the number of epochs, which refers to the number of iterations required to learn all the data, was 2000.

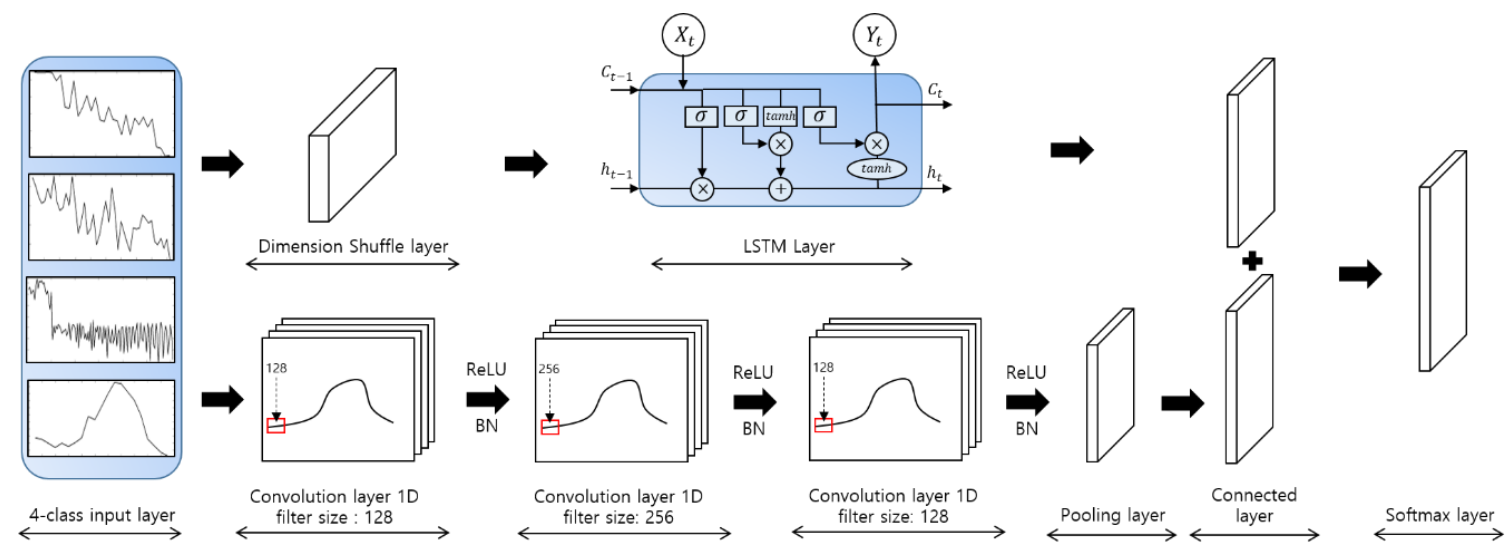

Figure 6. LSTM-FCN model for pet dog sound events classification.

The voltage time-series data represented as the four classes are converted from multivariate time-series data to single time step data by the dimension shuffle layer. The entire time-series data converted into a single time step are processed by the LSTM layer. Simultaneously, the same time-series data is shuffled through one-dimensional convolution layers with filter sizes of 128, 256, and 128 to perform fully convolutional network. This can be conducted in three steps, and the fully convolutional network of each step involves ReLU activation and batch normalization. By applying global average pooling, which outputs a feature map containing the reliability of the target class from the previous layer to the converted time-series data, the number of parameters of the network is reduced and the risk of overfitting is eliminated. The output values of the pooling layer and the LSTM layer are connected via the connected layer. Finally, the softmax is applied to allow for multiclass classification. At this time, the number of softmax layers is equal to the number of output layers.

Algorithm 1 shows the overall proposed method.

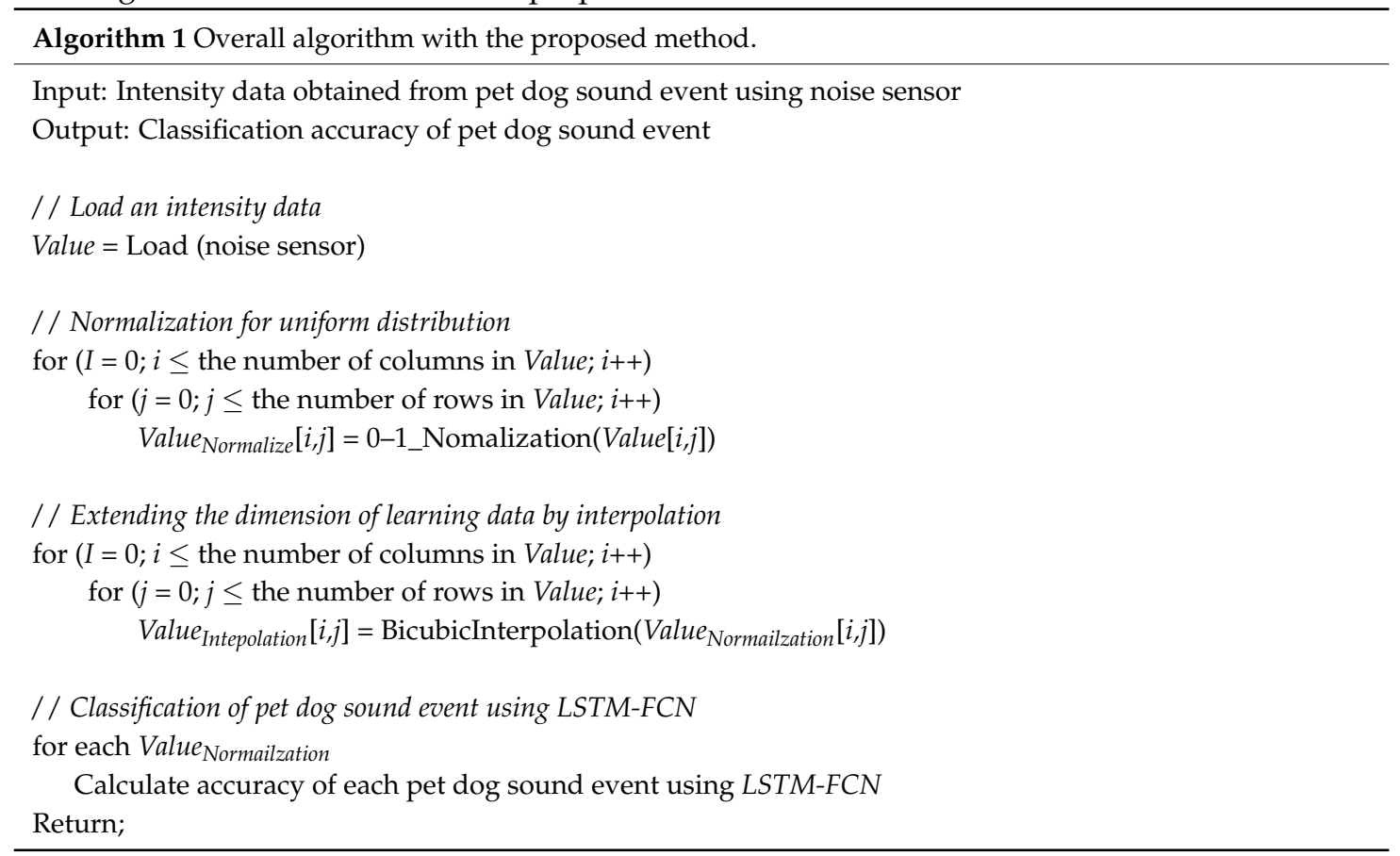




\section{Experimental Results}

\subsection{Experimental Environment}

We conducted experiments using a noise sensor to classify the sound events of dogs using a single PC. The CPU of the utilized PC was an Intel Core i7-7700K (8 cores; Intel, Santa Clara, CA, USA), the GPU was an NVIDIA GeForce GTX 1080Ti 11 GB (3584 CUDA cores; NVIDIA, Santa Clara, CA, USA) and the RAM size was 32 GB. We also used TensorFlow 1.8 in Ubuntu 16.04.2 (Canonical Ltd., London, UK) to implement the LSTM-FCN technique and experimented with Keras, an open-source neural network library written in Python 3.6.5.

To acquire intensity data in a wireless environment, a noise sensor was connected to an Arduino Pro Mini board. The Arduino Pro Mini is the smallest available Arduino board and offers similar functionality as the ATmega328 series found in the usual Uno board. Furthermore, it is available as a $5 \mathrm{~V} / 16 \mathrm{MHz}$ model and 3.3 V/8 MHz model, which differ in their operating voltage and have input voltages of 5-9 V and 3.3-9 V, respectively. Since the proposed method involved attaching the sensor to the neck of the dog, a LM-393 noise sensor was used in combination with the Arduino Pro Mini $5 \mathrm{~V} / 16 \mathrm{MHz}$. In addition, a Wi-Fi ESP8266 module was installed for wireless data transmission.

The intensity data acquired to classify the pet dog sound events were divided into four classes: barking, growling, howling, and whining. These were representative sounds produced by a pet dog in response to the stress of separation anxiety that can be felt by being isolated from the pet dog owner. Note that these sounds can also serve as an alert signal, or express fear in response to the presence of a stranger. Intensity data on the pet dog sounds were acquired via the attached noise sensor. The acquired intensity data were transmitted to the IoT analysis platform, which refined and processed the data.

The acquired data were classified according to four features, and each intensity data sample consisted of each sound event which was constituted as 300 datasets. For each feature, that is, the data generated from 300 sound events was labeled as one class, with a total of 1200 sound events. The pet dog sound events data sets are available in Supplementary Materials. Sampling of the acquired time-series data was performed at a rate of 138 samples per second, and thus we obtained a total of 88,617 samples.

Table 5 shows an example of intensity data for pet dog sound event obtained from noise sensor.

Table 6 shows the number of data for each class. Here, 5771 barking and 8390 whining events were acquired respectively due to their relatively short features. In addition, 17,877 growling and 56,579 howling events were obtained, respectively. 
Table 5. An example of intensity data for pet dog sound event obtained from noise sensor.

\begin{tabular}{cccccccccccccccccc}
\hline Pet Dog Sound Events & $\mathbf{1 / 1 3 8 ~ S e c ~}$ & & & & \multicolumn{1}{c}{ Intensity Level } \\
\hline & $1-16$ & 4.82 & 4.81 & 4.79 & 4.81 & 4.81 & 4.34 & 4.66 & 4.46 & 4.47 & 4.19 & 3.48 & 2.84 & 2.23 & 1.68 & 1.59 & 2.38 \\
Barking & $17-32$ & 2.84 & 3.62 & 4.34 & 3.92 & 2.97 & 2.31 & 2.28 & 2.54 & 2.82 & 3.09 & 3.38 & 3.50 & 3.26 & 2.85 & 2.63 & 2.84 \\
& $33-48$ & 2.23 & 1.68 & 1.59 & 2.38 & 3.62 & 4.34 & 3.92 & 2.97 & 2.31 & 2.28 & 2.54 & 2.82 & 3.09 & 3.38 & 3.50 & 3.26 \\
& $49-64$ & 2.85 & 2.63 & 2.84 & 3.23 & 3.4 & 3.07 & 2.52 & 1.94 & 1.91 & 2.16 & 2.22 & 2.55 & 2.85 & 3.00 & 3.11 & 3.33 \\
& $1-16$ & 4.45 & 4.28 & 3.83 & 2.99 & 2.49 & 2.63 & 3.11 & 3.54 & 3.80 & 4.01 & 4.04 & 3.73 & 3.26 & 2.93 & 2.94 & 3.11 \\
& $17-32$ & 3.17 & 2.96 & 2.64 & 2.37 & 2.14 & 1.96 & 2.03 & 2.58 & 3.37 & 3.91 & 3.85 & 3.52 & 3.39 & 3.74 & 4.28 & 4.55 \\
Growling & $33-48$ & 4.29 & 3.76 & 3.22 & 2.66 & 2.09 & 1.81 & 2.01 & 2.49 & 2.94 & 3.25 & 3.53 & 3.66 & 3.48 & 3.15 & 3.06 & 3.49 \\
& $49-64$ & 4.15 & 4.50 & 4.25 & 3.70 & 3.14 & 2.57 & 1.99 & 1.65 & 1.68 & 1.94 & 2.25 & 2.63 & 3.05 & 3.19 & 2.77 & 2.03 \\
\hline & $1-16$ & 3.61 & 4.08 & 4.28 & 4.15 & 3.95 & 3.52 & 2.64 & 2.18 & 1.95 & 1.88 & 2.01 & 2.47 & 3.13 & 3.58 & 3.56 & 3.32 \\
& $17-32$ & 3.19 & 3.31 & 3.54 & 3.75 & 3.88 & 3.99 & 4.03 & 3.92 & 3.75 & 3.65 & 3.77 & 2.55 & 2.15 & 2.62 & 2.57 & 2.08 \\
& $33-48$ & 2.48 & 3.30 & 3.89 & 3.97 & 3.81 & 3.06 & 2.48 & 2.20 & 2.54 & 3.18 & 3.54 & 3.27 & 2.73 & 2.37 & 2.38 & 2.57 \\
& $49-64$ & 2.80 & 3.11 & 3.45 & 3.50 & 2.93 & 2.08 & 1.55 & 1.71 & 2.19 & 2.50 & 2.35 & 2.03 & 1.82 & 1.85 & 2.00 & 2.11 \\
\hline & $1-16$ & 0.01 & 0.33 & 0.76 & 1.05 & 1.04 & 0.89 & 0.71 & 0.48 & 0.19 & 0.76 & 1.41 & 2.20 & 2.62 & 2.27 & 1.56 & 1.11 \\
& $17-32$ & 1.69 & 1.98 & 2.16 & 2.96 & 3.16 & 3.31 & 3.41 & 3.46 & 3.47 & 3.35 & 3.02 & 2.57 & 2.20 & 2.01 & 1.85 & 1.65 \\
& $33-48$ & 1.22 & 0.70 & 0.31 & 0.10 & 0.01 & 0.15 & 0.39 & 0.52 & 0.40 & 0.18 & 2.20 & 2.54 & 3.18 & 3.54 & 2.73 & 2.37 \\
& $49-64$ & 2.08 & 1.94 & 1.81 & 1.63 & 1.44 & 1.38 & 1.57 & 1.90 & 2.09 & 1.97 & 1.72 & 1.58 & 1.94 & 2.21 & 1.95 & 1.68 \\
\hline
\end{tabular}


Table 6. Number of data of four pet dog sound events.

\begin{tabular}{ccc}
\hline Pet Dog Sound Events & \# of Events & \# of Intensity Data per Event \\
\hline Barking & 300 & 5771 \\
Growling & 300 & 17,877 \\
Howling & 300 & 56,579 \\
Whining & 300 & 8390 \\
Total Number of Data & 1200 & 88,617 \\
\hline
\end{tabular}

\subsection{Comparison of Results Based on Sound and Intensity Data}

In this paper, four sound events (i.e., barking, growling, howling, and whining) of pet dogs were acquired using a noise sensor. After that, $0-1$ normalization was also applied to keep the distribution of data values constant. Then, we increased the lengths of the intensity data gradually with bicubic interpolation.

Figure 7 shows the visualization of each accuracy resulted in the LSTM-FCN model when the length of the datasets was increased through bicubic interpolation. The results show that the classification accuracy of the original data without bicubic interpolation is approximately $74 \%$. When the length of the data was increased by a factor of three, we confirmed that the classification accuracy with bicubic interpolation was $84 \%$. Note that the more length was increased than three times, the more classification accuracy was decreased.

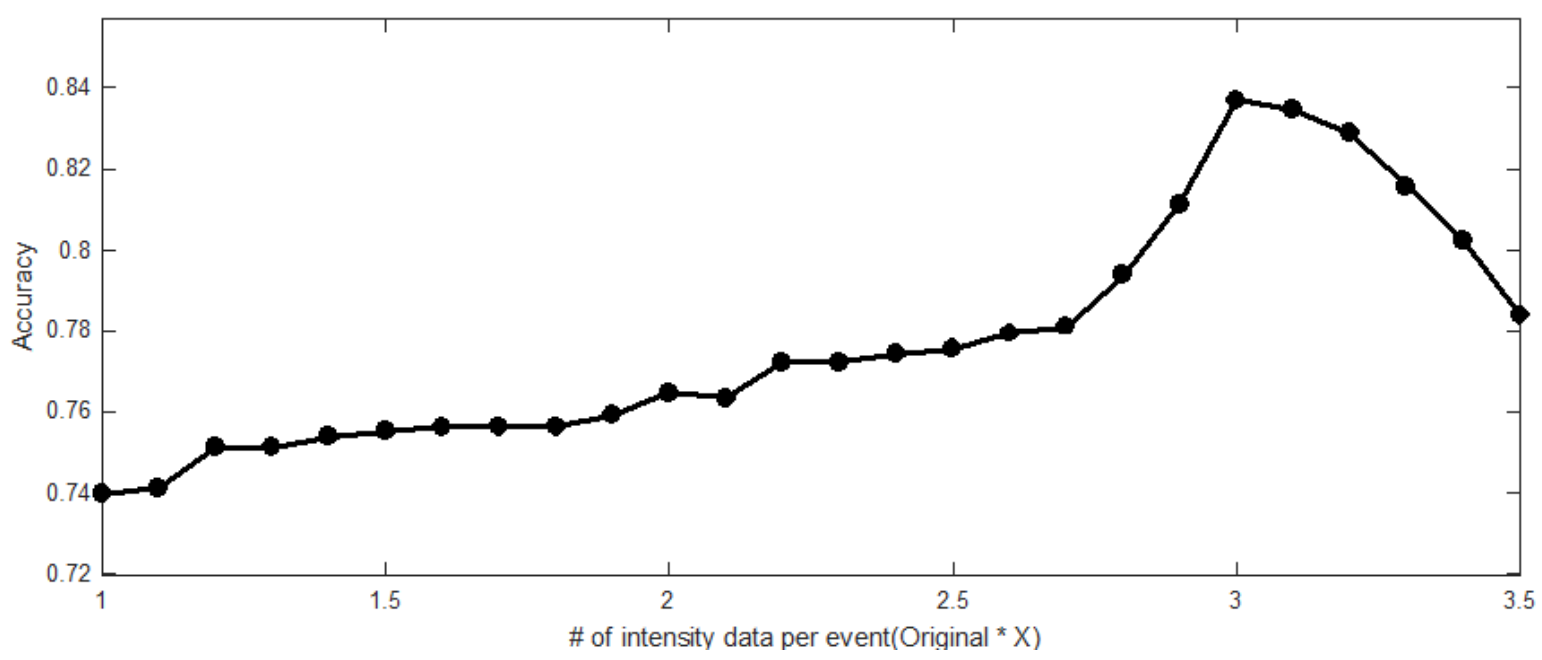

Figure 7. Each accuracy when increasing the data length through bicubic interpolation. The classification accuracy is improved if the length of the intensity data is increased compared to the original data. However, the classification accuracy is decreased if the increased length of the intensity data is exceeded three times compared to the length of the original data.

In order to evaluate the proposed method, we conducted a comparative experiment on the sound data recorded by the sound sensor. The sound data recorded for the experiment were acquired irrespective of the type and size of the pet dogs. The sound data were obtained from uncompressed WAV (waveform audio file) format, which can convert analog sounds into digital without data loss. The sampling rate of the sound data was $44,100 \mathrm{~Hz}$ using a mono channel, and the data were not affected significantly by ambient noise. The 1200 acquired samples had the same duration.

To compared to typical approaches based on sound analysis, we used three feature extraction methods (i.e., MFCC [28], spectrogram [29], and mel-spectrum [30]) and two classification methods (i.e., SVM [31] and K-NN [32]). Note that, since the intensity data is time series data, we applied the Shapelets [33] and LSTM-FCN [27] without the feature extraction methods. Note that, to extract the features, each the pet dogs sound corresponding to the interval of 1 to $3 \mathrm{~s}$ was separated manually. 
Table 7 compares each accuracy of using different features (MFCC, Spectrogram, and mel-spectrum) and classification methods (SVM, K-NN, Shapelet, LSTM-FCN, and LSTM-FCN with bicubic). To validate the proposed method, a comparative experiment was performed using the time-series methods (i.e., Shapelet, LSTM-FCN, and LSTM-FCN with bicubic) on the intensity data and the typical method (i.e., SVM and K-NN). The accuracies of the typical classification methods were approximately $78 \%$ to $86 \%$, versus approximately $74 \%$ for the LSTM-FCN and $84 \%$ for the LSTM-FCN with bicubic interpolation. These results confirm that the proposed method is suitable for classifying the sound events of a pet dog. Although, the proposed method achieves relatively low accuracy compared to the typical methods, it manages to attain a high gain in energy efficiency, including data size and power consumption without degradation of significant accuracy.

Table 7. Classification accuracy applied to each model.

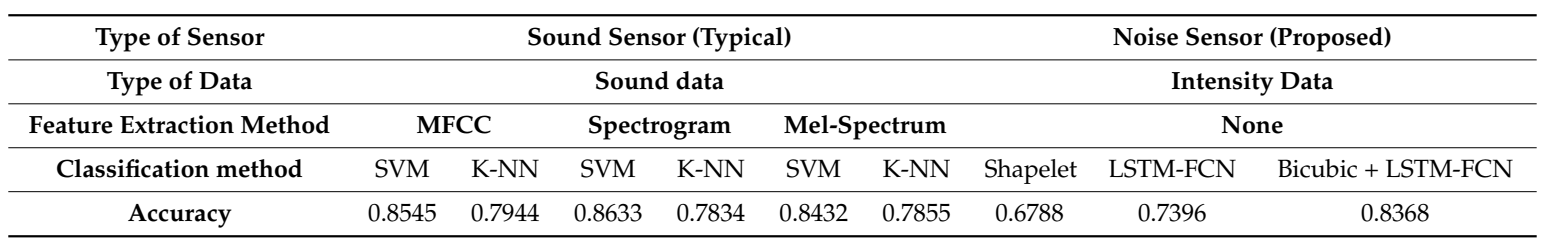

Table 8 lists the data size and power consumption of the sound sensor, noise sensor, and Wi-Fi sensor used in the experiment. The power of the noise sensor represents the sum of the power of the Arduino Pro Mini and the LM-393 sensor.

Table 8. Comparison of data size and performance between sound sensor and noise sensor, and Wi-Fi sensor.

\begin{tabular}{ccccc}
\hline Type of Device & Average of Data Size (KB) & Current (mA) & Voltage (V) & Energy (J) \\
\hline Sound sensor (MQ-U300) & 66.4 & 180 & 5.0 & 0.9 \\
Noise sensor (LM-393) & 0.9 & 20 & 5.0 & 0.1 \\
Wi-Fi (ESP8266) & - & 170 & 3.3 & 0.5 \\
\hline
\end{tabular}

With regard to the average data size, the sound data sensor performs relatively poorly due to the nature of the WAV format, which uses no compression. Therefore, the intensity data achieves a value approximately 73.8 times better than that of the sound data in this regard. In addition, the supply voltage of the sound sensor and the noise sensor used in the experiment is $5 \mathrm{~V}$, which means that the same voltage value is applied to both. The difference in current can be attributed to differences in overall resource efficiency.

In addition, the proposed method utilizes a system whose efficiency is sensitive to the battery usage time. The efficiency, with respect to battery usage time, is calculated based on the capacity of a Li-ion battery installed in a typical wearable device such as a smart watch. To date, no smart watch has been released that exceeds $400 \mathrm{mAh}$. This is one of the disadvantages of wearable devices that result from miniaturization. For example, when the battery capacity was $400 \mathrm{mAh}$, and the voltage was $5 \mathrm{~V}$, the total amount of electrical energy was $7200 \mathrm{~J}$. Since the sensing data has to be transmitted to the IoT platform, the transmission energy consumption should be also considered. To calculate the transmission energy consumption, we used $802.11 \mathrm{~b}$, which was supported by Wi-Fi sensor. The $802.11 \mathrm{~b}$ standard technology has a theoretical maximum transmission rate of $11 \mathrm{Mbps}$, and supports a transmission speed of about 6 to $7 \mathrm{Mbps}$ in the implementation of CSMA/CA technology. Therefore, we used network conditions with $300 \mathrm{~KB} / \mathrm{s}$ to $1200 \mathrm{~KB} / \mathrm{s}$ as shown in Table 7. To calculate the total energy consumption, we considered both the sensing and transmission energy consumption. Note that, the transmission energy consumption depends on the network conditions. Since the sound sensing data required lager transmission data size than noise sensing data, the transmission energy consumption was also more required. 
The sensing energy consumption of sound and noise was $0.9 \mathrm{~J}$ and $0.1 \mathrm{~J}$ for one second with various network conditions, respectively, and the transmission energies of 0.111 to $0.028 \mathrm{~J}$ and 0.002 to $0.001 \mathrm{~J}$ were obtained. Finally, by calculating the battery usage time (i.e., battery capacity was $400 \mathrm{~mA}$ ), we found out that the sound sensor can be used for about $1.9 \mathrm{~h}$, and the noise sensor can be used for $19.6 \mathrm{~h}$. Therefore, we confirmed that the proposed method (i.e., with noise sensor) can improve the energy efficiency about 10 times than the typical method (i.e., with sound sensor). Table 9 shows that sensing, transmission, total energy consumption for one second, and battery usage time with various network conditions (i.e., 300, 600, 900, and $1200 \mathrm{~KB} / \mathrm{s}$ ).

Table 9. Comparison of energy consumption and battery usage with various network conditions.

\begin{tabular}{cccccc}
\hline \multirow{2}{*}{} & & \multicolumn{4}{c}{ Transmission Speed (KB/s) } \\
\cline { 3 - 6 } & & $\mathbf{3 0 0}$ & $\mathbf{6 0 0}$ & $\mathbf{9 0 0}$ & $\mathbf{1 2 0 0}$ \\
\hline \multirow{2}{*}{ Sensing Energy (J) } & Sound & \multicolumn{4}{c}{0.9} \\
& Noise & & \multicolumn{2}{c}{0.1} & \\
& Sound & 0.111 & 0.056 & 0.037 & 0.028 \\
\multirow{2}{*}{ Transmission Energy (J) } & Noise & 0.002 & 0.001 & 0.001 & 0.001 \\
& Sound & 1.011 & 0.956 & 0.937 & 0.928 \\
& Noise & 0.102 & 0.101 & 0.101 & 0.101 \\
\hline \multirow{2}{*}{ Total Energy (J) } & Sound & 1.9 & 2.0 & 2.1 & 2.2 \\
\multirow{2}{*}{ Battery usage time (h) } & Noise & 19.6 & 19.8 & 19.8 & 19.8 \\
\hline
\end{tabular}

\section{Conclusions}

The classification of pet dog sound events using data from a sound sensor is important for analyzing the behavior or emotions of pet dogs that are left alone. In this paper, we proposed a way to classify pet dog sound events (barking, growling, howling, and whining) to improve resource efficiency without significant degradation of accuracy. We acquired intensity data from pet dog sound events using a relatively resource-efficient noise sensor instead of a sound sensor. Generally, it is difficult to achieve sufficient classification accuracy using the intensity of sound, due to the loss of information in the sound data. To avoid significant degradation of classification accuracy, we applied LSTM-FCN, and exploited bicubic interpolation. Based on the experimental results, which found the typical methods to be $78 \%$ to $86 \%$ accurate and the proposed method to be $84 \%$ accurate, we can confirm that the proposed method based on noise sensor based on noise sensor (i.e., Shapelet and LSTM-FCN for time-series) improved energy efficiency by 10 times without significant degradation of accuracy compared to typical methods based on sound sensor (i.e., MFCC, Spectrogram, and mel-spectrum for feature extraction, and SVM and K-NN for classification).

Supplementary Materials: The pet dog sound events data sets are available online at https://github.com/ kyb2629/pdse.

Author Contributions: Y.C., D.P. and S.L. conceived and designed the overall analysis model; Y.K. and J.S. collected sound data and intensity data; Y.K., J.S. and S.L. analyzed the experimental results; Y.K., J.S., Y.C., D.P. and S.L. wrote the paper.

Funding: This research received no external funding.

Acknowledgments: This study was supported by a Korea University Grant.

Conflicts of Interest: The authors declare no conflict of interest.

\section{References}

1. Chung, Y.; Lee, S.; Jeon, T.; Park, D. Fast Video Encryption Using the H. 264 Error Propagation Property for Smart Mobile Devices. Sensors 2015, 15, 7953-7968. [CrossRef] [PubMed]

2. Lee, S.; Jeong, T. Forecasting Purpose Data Analysis and Methodology Comparison of Neural Model Perspective. Symmetry 2017, 9, 108. [CrossRef] 
3. Lee, S.; Kim, H.; Chung, Y.; Park, D. Energy Efficient Image/video Data Transmission on Commercial Multi-core Processors. Sensors 2012, 12, 14647-14670. [CrossRef] [PubMed]

4. Lee, S.; Kim, H.; Sa, J.; Park, B.; Chung, Y. Real-time Processing for Intelligent-surveillance Applications. IEICE Electron. Express 2017, 14, 20170227. [CrossRef]

5. Lee, S.; Jeong, T. Cloud-based Parameter-driven Statistical Services and Resource Allocation in a Heterogeneous Platform on Enterprise Environment. Symmetry 2016, 8, 103. [CrossRef]

6. Ribeiro, C.; Ferworn, A.; Denko, M.; Tran, J. Canine Pose Estimation: A Computing for Public Safety Solution. In Proceedings of the 2009 Canadian Conference on Computer and Robot Vision, Kelowna, BC, Canada, 25-27 May 2009; pp. 37-44.

7. Pongrácz, P.; Molnár, C.; Miklósi, Á. Barking in Family Dogs: An Ethological Approach. Vet. J. 2010, 183, 141-147. [CrossRef] [PubMed]

8. Chung, Y.; Lee, J.; Oh, S.; Park, D.; Chang, H.H.; Kim, S. Automatic Detection of Cow's Estrus in Audio Surveillance System. Asian-Australas. J. Anim. Sci. 2013, 26, 1030-1037. [CrossRef] [PubMed]

9. Ye, L.; Keogh, E. Time Series Shapelets: A New Primitive for Data Mining. In Proceedings of the 15th ACM SIGKDD International Conference on Knowledge Discovery and Data Mining, Paris, France, 28 June-1 July 2009; pp. 947-956.

10. Karim, F.; Majumdar, S.; Darabi, H.; Chen, S. LSTM Fully Convolutional Networks for Time Series Classification. IEEE Access 2018, 6, 1662-1669. [CrossRef]

11. Lukman, A.; Harjoko, A.; Yang, C.K. Classification MFCC Feature from Culex and Aedes Aegypti Mosquitoes Noise Using Support Vector Machine. In Proceedings of the 2017 International Conference on Soft Computing, ICSIIT, Denpasar, Indonesia, 26-28 September 2017; pp. 17-20.

12. Zhang, W.; Han, J.; Deng, S. Heart sound classification based on scaled spectrogram and partial least squares regression. Biomed. Signal Process. Control 2017, 32, 20-28. [CrossRef]

13. Dong, M. Convolutional Neural Network Achieves Human-level Accuracy in Music Genre Classification. arXiv, 2018; arXiv:1802.09697.

14. Sonawane, A.; Inamdar, M.U.; Bhangale, K.B. Sound based human emotion recognition using MFCC \& multiple SVM. In Proceedings of the International Conference on Information, Communication, Instrumentation and Control (ICICIC), Indore, India, 17-19 August 2017; pp. 1-4.

15. Kim, J.; Park, C.; Ahn, J.; Ko, Y.; Park, J.; Gallagher, J.C. Real-time UAV sound detection and analysis system. In Proceedings of the 2017 IEEE Sensors Applications Symposium (SAS), Glassboro, NJ, USA, 13-15 March 2017; pp. 1-5.

16. Khwarahm, N.R.; Dash, J.; Skjøth, C.A.; Newnham, R.M.; Adams-Groom, B.; Head, K.; Caulton, E.; Atkinson, P.M. Mapping the Birch and Grass Pollen Seasons in the UK Using Satellite Sensor Time-series. Sci. Total Environ. 2017, 578, 586-600. [CrossRef] [PubMed]

17. Vitola, J.; Pozo, F.; Tibaduiza, D.A.; Anaya, M. A Sensor Data Fusion System Based on K-nearest Neighbor Pattern Classification for Structural Health Monitoring Applications. Sensors 2017, 17, 417. [CrossRef] [PubMed]

18. Liu, J.; Fu, Y.; Ming, J.; Ren, Y.; Sun, L.; Xiong, H. Effective and Real-time In-app Activity Analysis in Encrypted Internet Traffic Streams. In Proceedings of the 23rd ACM SIGKDD International Conference on Knowledge Discovery and Data Mining, Halifax, NS, Canada, 13-17 August 2017; pp. 335-344.

19. Chen, Z.; He, K.; Li, J.; Geng, Y. Seq2Img: A Sequence-to-image Based Approach towards IP Traffic Classification Using Convolutional Neural Networks. In Proceedings of the 2017 IEEE Conference on Big Data, Boston, MA, USA, 11-14 December 2017; pp. 1271-1276.

20. Zhang, Y.; Pezeshki, M.; Brakel, P.; Zhang, S.; Bengio, C.L.Y.; Courville, A. Towards End-to-end Speech Recognition with Deep Convolutional Neural Networks. arXiv, 2017; arXiv:1701.02720.

21. Pei, W.; Dibeklioğlu, H.; Tax, D.M.; van der Maaten, L. Multivariate Time-series Classification Using the Hidden-unit Logistic Model. IEEE Trans. Neural Netw. Learn. Syst. 2018, 29, 920-931. [CrossRef] [PubMed]

22. Soares, E.; Costa, P., Jr.; Costa, B.; Leite, D. Ensemble of Evolving Data Clouds and Fuzzy Models for Weather Time Series Prediction. Appl. Soft Comput. 2018, 64, 445-453. [CrossRef]

23. Manandhar, S.; Dev, S.; Lee, Y.H.; Meng, Y.S.; Winkler, S. A Data-driven Approach to Detecting Precipitation from Meteorological Sensor Data. arXiv, 2018; arXiv:1805.01950. 
24. Hu, Y.; Gunapati, V.Y.; Zhao, P.; Gordon, D.; Wheeler, N.R.; Hossain, M.A.; Peshek, T.J.; Bruckman, L.S.; Zhang, G.Q.; French, R.H. A Nonrelational Data Warehouse for the Analysis of Field and Laboratory Data from Multiple Heterogeneous Photovoltaic Test Sites. IEEE J. Photovolt. 2017, 7, 230-236. [CrossRef]

25. Garcke, J.; Iza-Teran, R.; Marks, M.; Pathare, M.; Schollbach, D.; Stettner, M. Dimensionality Reduction for the Analysis of Time Series Data from Wind Turbines. In Proceedings of the Scientific Computing and Algorithms in Industrial Simulations, Cham, Switzerland, 19 July 2017; pp. 317-339.

26. Wilson, S.J. Data Representation for Time Series Data Mining: Time Domain Approaches. WIREs Comput. Stat. 2017, 9, e1392. [CrossRef]

27. Egri, A.; Horváth, I.; Kovács, F.; Molontay, R.; Varga, K. Cross-correlation Based Clustering and Dimension Reduction of Multivariate Time Series. In Proceedings of the 2017 IEEE 21st International Conference on Intelligent Engineering Systems, Larnaca, Cyprus, 20-23 October 2017; pp. 241-246.

28. Um, T.T.; Pfister, F.M.; Pichler, D.; Endo, S.; Lang, M.; Hirche, S.; Fietzek, U.; Kulić, D. Data Augmentation of Wearable Sensor Data for Parkinson's Disease Monitoring Using Convolutional Neural Networks. In Proceedings of the 19th ACM International Conference on Multimodal Interaction, New York, NY, USA, 13-17 November 2017; pp. 216-220.

29. Salamon, J.; Bello, J.P. Deep Convolutional Neural Networks and Data Augmentation for Environmental Sound Classification. IEEE Signal Process. Lett. 2017, 24, 279-283. [CrossRef]

30. Feng, Z.H.; Kittler, J.; Christmas, W.; Huber, P.; Wu, X.J. Dynamic Attention-controlled Cascaded Shape Regression Exploiting Training Data Augmentation and Fuzzy-set Sample Weighting. In Proceedings of the 2017 IEEE Conference on Computer Vision and Pattern Recognition, Honolulu, HI, USA, 21-26 July 2017; pp. 3681-3690.

31. Krogh, A.; Larsson, B.; Von Heijne, G.; Sonnhammer, E.L. Predicting Transmembrane Protein Topology with a Hidden Markov Model: Application to Complete Genomes. J. Mol. Biol. 2001, 305, 567-580. [CrossRef] [PubMed]

32. Berndt, D.J.; Clifford, J. Using Dynamic Time Warping to Find Patterns in Time Series. In Proceedings of the KDD Workshop, Seattle, WA, USA, 31 July-1 August 1994; pp. 359-370.

33. Adafruit. Measuring Sound Levels. Available online: https://learn.adafruit.com/adafruit-microphoneamplifier-breakout/measuring-sound-levels (accessed on 6 July 2018).

(C) 2018 by the authors. Licensee MDPI, Basel, Switzerland. This article is an open access article distributed under the terms and conditions of the Creative Commons Attribution (CC BY) license (http:/ / creativecommons.org/licenses/by/4.0/). 